\title{
Pratiques sacrificielles chez les Mitsogho du Gabon
}

Sacrificial Practices among the Tsogo (Gabon)

Otto Gollnhofer et Roger Sillans

\section{(2) OpenEdition}

Journals

Édition électronique

URL : http://journals.openedition.org/span/626

DOI : $10.4000 /$ span. 626

ISSN : 2268-1558

Éditeur

École pratique des hautes études. Sciences humaines

Édition imprimée

Date de publication : 1 septembre 1986

Pagination : 155-174

ISSN : 0294-7080

\section{Référence électronique}

Otto Gollnhofer et Roger Sillans, "Pratiques sacrificielles chez les Mitsogho du Gabon », Systèmes de pensée en Afrique noire [En ligne], 7 | 1986, mis en ligne le 05 juin 2013, consulté le 01 mai 2019. URL: http://journals.openedition.org/span/626 ; DOI : 10.4000/span.626 


\title{
PRATIQUES SAGRIFICIELLLES \\ CHEZ LES MITSOGHO DU GABON
}

\author{
par \\ Otto Gollnhofer \& Roger Sillans
}

Les opérations de type sacrifiel chez les Mitsogho font partie d'un ensemble de pratiques religieuses visant à manipuler des forces invisibles placées sous le contrôle d'ancêtres ou de génies. Le déclenchement du processus sacrificiel suppose l'intervention d'entités que l'on sollicite d'intervenir soit par le truchement de certains objets ou de certaines parties du corps humain ou d'animaux (plumes d'aigle ou de perroquet, bec d'oiseaux, dépouilles et dents de certains animaux), soit par celui de substances diverses choisies en raison de leurs rapports d'analogie (1) avec les fins que l'on se propose d'atteindre. Cet ensemble (objets et substances diverses) constitue ce que nous appelerons "l'instrumentalité sacrificielle". L'intervention de ces entités se manifeste par le biais d'objets-médiateurs qui deviennent le siège transitoire de leur "émanation". Quel que soit le but à atteindre bénéfique ou maléfique -, le mécanisme se déclenche toujours de la même façon. L'émanation de l'entité spirituelle, qui a investi l'objet-médiateur à la demande de I'officiant, quitte cet objet, sur l'ordre de 1 'opérateur, pour accomplir sa mission ; après quoi elle réintègre son support d'origine. Cette même opération doit être renouvelée à maintes reprises en tenant compte, toutefois, du fait que la force qui anime ce siège se perd avec le temps. Elle peut même se retourner contre son détenteur ou contre la communauté au nom de laquelle le sacrifice a été consacré. Les aīeuls (koko) de la famille du détenteur de l'objet-médiateur détruisent alors celui-ci par le feu et jettent les résidus dans la rivière la plus proche du village. Au retour, la famille absout la personne qui détenait cet objet, au cours d'une cérémonie publique.

Systèmes de pensée, Cahier 7, 1984 
Nous avons déjà eu 1 'occasion de montrer que la conception mitsogho de 1 'activité rituelle est centrée autour de la notion de maghangha, qu'on peut traduire dans certains contextes par le terme de médecine (ou médicament), mais dont la signification déborde largement le champ sémantique couvert par ce terme (2). Les activités liées à 1a manipulation des mighanga englobe les pratiques cultuelles collectives de toutes les sociétés et confréries initiatiques des deux sexes ainsi que celles s'exerçant individuellement dans le cadre privé ou public.

Dans une précédente communication (3), nous avons donné un aperçu général du sacrifice tel qu'on peut le saisir à travers l'étude des mythes et des rituels initiatiques. Nous nous proposons ici de décrire quelques pratiques sacrificielles effectuées dans le contexte des relations rituelles que les Mitsogho entretiennent avec leurs morts. Nous verrons que ces rites n'impliquent pas nécessairement une mise à mort sanglante d'animaux.

Sacrifices funéraires

Les Mitsogho ont plusieurs sociétés initiatiques et chacune de ces sociétés a ses rites funéraires propres. Vers 1'âge de 10 ans, un individu de sexe masculin appartient normalement à plusieurs d'entre elles. Il en résulte que lorsque survient le décès d'un homme adulte, sa famille doit choisir le type de funérailles à accomplir. Nous envisagerons successivement les sacrifices effectués auprès de la tombe dans le cadre des funérailles organisées par la société du Bwete (la société la plus importante de l'ethnie tsogho) d'une part, dans celui de la société du Ya-Mwei (société consacrée au culte du génie de la terre et de l'eau) d'autre part.

Dans les dernières séquences des rites funéraires de type Bwete, les initiés se regroupent dans la maison du culte, ebanza, située dans le village. Cet édifice se présente comme une construction rectangulaire ouverte sur 1 'un de ses côtés (cf.photo \& fig.1). De chaque côté de l'entrée se dresse une colonnette sculptée représentant respectivement un ancêtre mâle (côté droit) et un ancêtre femelle (côté gauche). Ces deux colonnettes soutiennent 1'avant-tô̂t de l'ebanza. 


$$
\text { [.] }
$$


Une colonne centrale sur laquelle est sculptée le plus souvent une figure anthropomorphe maintient 1a partie antérieure du faîtage de 1 'ensemble de la construction. Pendant l'exécution des funérailles, on doit installer dans l'ebanza un objet rituel spécifique, sorte de cloison de feuillage dite ndembe (cf. Fig. 1). Une fois entrés dans l'ebanza, les initiés se disposeront de part et d'autre de cette cloison. D'un côté se placeront diverses catégories de musiciens, de 1'autre, dissimulés au regard des premiers, viendront prendre place ceux des initiés qui sont chargés de représenter diverses entités spirituelles. A travers les paroles et les sons émis par ces initiés, c'est la voix des puissances qui se manifeste. C'est ainsi que l'initié représentant Nzondo, le génie de la brousse, éméttra des sons très aigus et sifflants. L'initié incarnant Ya-Mwei (entité qui n'intervient pas seulement dans la société qu'elle préside et à laquelle elle a donné son nom, mais aussi dans 1e Bwete) émettra des sons graves et caverneux. Le ndembe sera ensuite transporté par les initiés sur les lieux de 1 'inhumation, situés en brousse, à 1 'extrémité du village. Un cortège se formera, dirigé par les parents les plus âgés du disparu. Arrivé au bout du village, on éteindra les torches et les initiés se débarrasseront du ndembe en le jetant au pied de certains végétaux (4). Parfois, le ndembe sera plongé dans un marigot. Dans les deux cas, on immolera un coq en versant son sang sur l'objet. Après avoir craché sur le ndembe du champignon kuna (5) longuement mâché, on s'adressera au défunt dans les termes suivants : "Nous ne voulons pas que notre clan ait des accidents, des morts ou des maladies !". A ce moment, un initié simulant Ghepobwe (entité qui est le symbole de la mort physique), fera entendre un long hurlement en brousse et les villageois devront alors se réfugier dans leurs demeures. Pendant qu'il criera, d'autres initiés abattront un parasolier dont 1a chute produira un fracas tel qu'on 1 'entend sur plusieurs kilomètres.

Des mises à mort interviennent également lors des funérailles d'un membre de la société Ya-Mwei et lors d'une cérémonie spéciale destinée à intégrer le défunt dans le monde de l'au-delà. Dans ce cas, avant d'aller en brousse, les initiés se rendront dans la cour du village, avec un coq rouge et un coq noir. Les rites publics villageois se dérouleront également dans la maison de l'ebanza qui, pour la circonstance, aura été spécialement aménagée : devant l'ebanza, on aura 
disposé une sorte de cloison semi-circulaire, appelée gheveva, d'une hauteur de deux à trois mètres, en feuilles de bananier (cf. Fig. 2). Dans I'espace délimité par cette cloison, sur l'axe médian, à mi-chemin entre 1 'entrée et le poteau central, un trou aura été creusé par les initiés, symbolisant la matrice de Ya-Mwei. C'est là que prennent place les deux ou trois initiés qui tiennent le rôle du génie Ya-Mwei. A l'extérieur de cette enceinte, dans le prolongement de l'axe médian de 1 'édifice, marqué à l'intérieur par la colonne centrale, se dresse l'arbre tsindo (Randia acuminata Bth, Rubiacées), fiché en terre après avoir été déraciné de son lieu naturel (il est alors désigné par le terme kuta). Au pied de 1'arbre, on aura attaché le coq rouge.

Dès le début du rituel, les initiés déposeront dans l'orifice de la poudre tsingo (poudre obtenue à partir du tronc de l'arbre de l'espèce padouk, Pterocarpus soyauxil Tauber, Papilionacées, dit aussi "bois rouge"), deux petits bâtonnets dits kondoko (1'un symbolise 1a mort, l'autre la vie), de la cire noire d'abeille sauvage (mungu) ainsi que les ongles des pattes droites des deux coqs. Un initié devra égorger le coq noir avec ses dents, laissant couler le sang dans le trou qui sera ensuite comblé avec des morceaux du champignon kuna. Alors 1 'adepte faisant office d"interprète" (mondonga) de Ya-Mwei crachera encore du Kuna dans cet orifice, puis prononcera les paroles suivantes à l'adresse du défunt : "Ne nous crée pas d'histoires ! Ne reviens pas sans qu'on te sollicite".

Les rites publics villageois une fois accomplis, les initiés du Ya-Mwei se rendront en brousse. Ils emporteront la corde de liane (6) - cette corde aura servi à secouer 1 'arbre kuta au moment où Ya-Mwei parlait à travers la bouche de son "interprète" - ainsi qu'une peau de genette (mosingi) (Genetta servalina servalina Pucheran, Carnivores), le tambour de Ya-Mwei (confectionné dans un tronc de bananier évidé), une torche allumée et le coq rouge vivant. Une fois parvenu devant le palmier nain (Phenix reclinata Jacq., Palmacées) qui représente la parturiente, un adepte, incarnant Ya-Mwei, creusera un trou, sorte de reduplication du trou creusé devant 1'ebanza qui, rappelons-le, symbolise la matrice de Ya-Mwei. Il le remplira des mêmes substances que celles décrites plus haut auxquelles il ajoutera deux petits paquets de feuilles, dénommés muenge. Ceci fait, il obturera l'orifice en y écrasant la torche allumée. Ce geste, pour les Mitsogho, signifie que le mort est définitivement "enfermé dans le ventre" de Ya-Mwei jusqu'au 


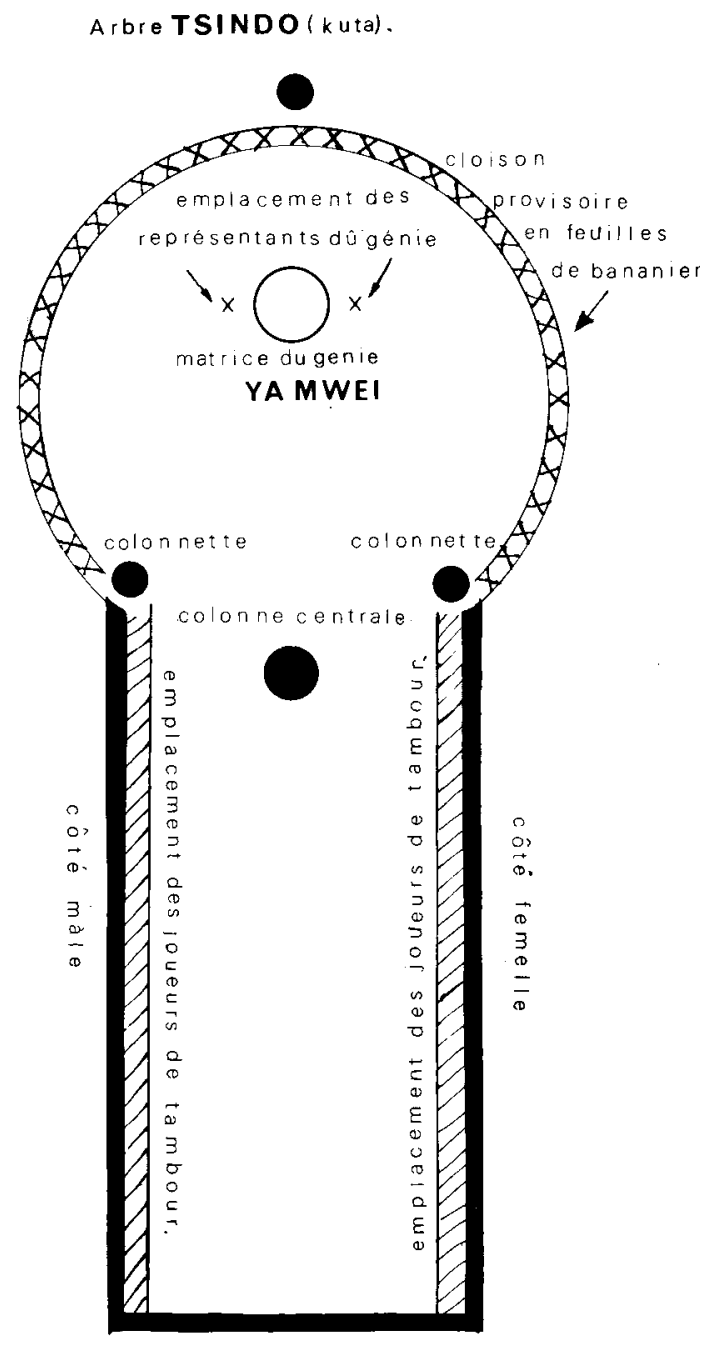

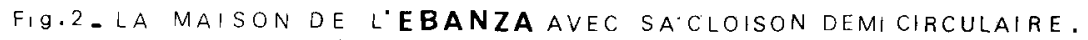


prochain cycle de réincarnation. Se servant d'un coq comme instrument de "purification", l'adepte se frottera ensuite le visage, le torse et l'abdomen pour faire disparâtre le fard rituel tsingo dont il s'était préalablement enduit. Il effacera ainsi le sang dont tout nouveau-né est recouvert à la naissance, plaçant donc une nouvelle fois le rituel de brousse sous le signe de la mort.

Les rites suivants auront pour finalité de rompre définitivement les liens qui rattachent encore le mort à la communauté des vivants.

L'adepte incarnant $\mathrm{Y}_{\boldsymbol{a}}-\mathrm{Mw}_{\mathrm{w}} \mathrm{i}$ cassera de la canne à sucre noire dite ghembimbi (variété de canne à sucre à type glabre, violet-noirâtre) en plusieurs petits morceaux en se servant de la petite enclume piriforme motendo. Alors chaque initié se frotte le visage et la poitrine avec le suc de cette canne, dont les morceaux sont déposés au pied du palmier. Puis l'officiant principal, représentant Ya-Mwei, crache sur cet arbre, dépose du charbon de bois (mbii) sur le trou et y enfonce deux petits bâtonnets kondoko. Ce même officiant commence alors à déplumer le coq vivant (le coq rouge) tout en récitant des textes rituels ; il interrompt parfois son discours, continue à déplumer la volaille, reprend la parole, s'arrête à nouveau, et ainsi de suite jusqu'au déplumage complet de 1 'animal. Il achévera de combler le trou avec les plumes de la volaille sacrifiée; il le recouvrira ensuite de la peau de genette. Précisons que c'est sur une peau de genette que les néophytes auront pris place au cours des rites de passage.

Le rite sera clôturé de la manière suivante. On enroulera autour du palmier nain la corde qui aura servi à secouer 1'arbre kuta. On placera auprès du même arbre une petite corbeille en rotin. Enfin, les initiés détruiront la cloison de feuillage gheveva et jetteront ses débris en brousse. Alors ils pourront réintégrer leur demeure.

Sacrifices liés aux crânes des ancêtres

Les Mitsogho vivent dans un univers hostile et plein de puissances maléfiques contre lesquelles ils mobilisent en permanence divers objets-médiateurs. Ces objets perdent périodiquement leur efficace et il est nécessaire soit de les remplacer soit de les recharger de force 
protectrice. C'est dans ce contexte de lutte et de protection contre ces forces maléfiques que les nganga (devins-guérisseurs bien connus dans le monde bantu) peuvent prescrire aux villageois d'aller exhumer le crâne d'un de leurs parents (il s'agit presque toujours d'un parent récemment décédé qui, avant de mourir, a donné sa bénédiction à ses descendants). Les nganga peuvent faire de pareilles prescriptions dans d'autres contextes, notamment dans des situations ( 1 a chasse par exemp1e) où il s'agit de se rendre propices les forces de la brousse.

Parfois, le crâne qui vient d'être exhumé fait l'objet d'un traitement rituel complexe. C'est le cas notamment lorsqu'il s'agit d'écarter une menace de malédiction ou d'inhiber une puissance hostile, ou encore d'utiliser une force occulte dans un but maléfique. Au moment de l'exhumation, on précisera au crâne le but à atteindre (ainsi, dans le cas d'un conflit intra-familial, on lui dira : "il faut que tu tues telle ou telle personne de notre famille; elle va te remplacer dans la tombe !"). Le crâne, d'abord frotté avec le miel ebonga et de 1 'huile de njave (Mimisops njave, Engler, Sapotacées), est ensuite saupoudré avec du tsingo (Pterocarpus soyauxii, Tauber, Papilionacées). Puis du champignon kuna mâché est craché sur le crâne, un oeuf de poule est cassé dans la cavité bucale et une plume du perroquet ngoso (Psittacus erithacus erithacus linné) est disposée sur l'os frontal. De la nourriture pour le mort (têtards, cassada, etc.) est mise sur des feuilles d'eembembe (Piper umbelzatum L., Pipéracées), placées devant le crâne qui est, finalement, enveloppé dans un pagne noir ebundi. En revenant de la tombe, les officiants chantent en passant dans la cour du village : "le propriétaire du village se promène, les pieds sales et gonflés ! Il se promène dehors". Le crâne est d'abord déposé au pied de la colonne centrale de la maison du culte ebanza, pour être placé ensuite au fond de l'édifice. Ces rites sont suivis de quatre cérémonies nocturnes accomplies dans le cadre de la société initiatique du Bwete. Après quoi le crâne est rangé définitivement dans 1a "case à médicaments" du Bwete, lieu oì l'on entrepose certains objets rituels notamment des crânes d'hommes et d'animaux ainsi que la statuaire prophylactique du village.

Parfois le rite fait intervenir des gheonga, statuettes ou bustes d'ancêtres qu'on place au sommet des crânes recouverts d'un pagne noir ebundi. Appelé mbumba, cet ensemble constitue l'un des plus puissants objets-médiateurs. La puissance liée au mbumba fait connaître 
par des rêves la nature des offrandes qu'il désire. Les présents offerts le plus fréquemment consistent en têtards, cassada, champignon kuna, huile de njave, offrandes par excellence des ancêtres. C'est toujours l'aĩeul de la famille qui est chargé de présenter ces offrandes.

Des formes de sacrifices faites aux crânes d'ancêtres sont également prescrites par le nganga pour rendre la chasse fructueuse (7). Le chasseur doit exhumer le crâne de 1'un de ses ancêtres proches et doit aussi se procurer le crâne d'un animal tué à la chasse. Il placera ces crânes dans sa maison en un endroit dit monumbu et, pendant la nuit, il posera dessus une série de cordes dans lesquelles, symboliquement, les animaux viendront se piéger. La veille du jour où il ira en brousse poser des pièges réels, on procédera au rite suivant : à l'extérieur de la maison, un groupe de femmes exécutera la danse liée aux génies de la brousse dits mighesi. Pendant ce temps, le piégeur, à l'intérieur de son habitation, prendra une demi-calebasse, et s'en servant comme d'une caisse de résonance, imitera la voix de ces génies, que les participants situés à 1 'extérieur reprendront en choeur. Le lendemain matin, le piégeur déposera du sang de gibier sur le crâne ainsi que le coeur d'un animal de brousse: C'est ce crâne qui, devenu objet-médiateur, donnera son efficacité aux pièges posés par le chasseur.

Certains individus ne peuvent propitier directement leurs parents défunts car on estime qu'ils leur ont porté préjudice de leur vivant. Dans ce cas, on demande aux personnes qui ont fait fonction de fossoyeurs lors des rites d'inhumation d'aller présenter les offrandes. La composition de l'offrande est la suivante : une poule, des têtards, des bananes pilées, de 1 'huile de njave et deux feuilles de eembembe. Après avoir nettoyé la tombe, un parent du défunt s'agenouille pour expliquer l'es raisons qui 1 'ont conduit à faire cette offrande. Alors on pose de chaque côté de la tombe une feuille de eembembe et sur chacune des feuilles, on place des têtard et des bananes pilées. Puis, à 1 'aide d'un morceau de bois qu'on enfonce en terre, on fait un conduit reliant la surface du sol au crâne du défunt. On incise la patte d'un coq et on en fait couler le sang ainsi que de l'huile de njave par 1 'orifice. Le coq est ensuite attaché à ce piquet de bois, fiché en terre devant la tombe ; près du coq, on place du taro. Les officiants s'éloignent ensuite de la tombe, et s'installent à environ 500 mètres de celle-ci. Ils resteront sur les lieux jusqu'à ce que le coq ait 
chanté, preuve que l'ancêtre a donné sa bénédiction. Alors les officiants retournent auprès de 1 a tombe, versent une seconde fois de 1 'huile de njave par l'orifice, emportant le coq ainsi que le taro jusqu'au village. Ils devront se rendre chez le parent du mort qui les a chargé d'accomplir ce "sacrifice" à leur place.

Dans les pages précédentes, nous avons décrit des rituels faisant intervenir dans des contextes divers des mises à mort d'animaux. Peut-on en toute rigueur définir ces traitements rituels comme des sacrifices ? Pourquoi les Mitsogho qui, en dehors de la volaille élèvent également des ovins et des caprins, n'en font-ils jamais usage pour "rentrer en communication" avec les différentes puissances de leur univers religieux ? Telles sont les questions que nous examinerons lors d'une prochaine étude.

Ot to Gollnhofer et R. Sillans

Chargés de Recherche au CNRS 


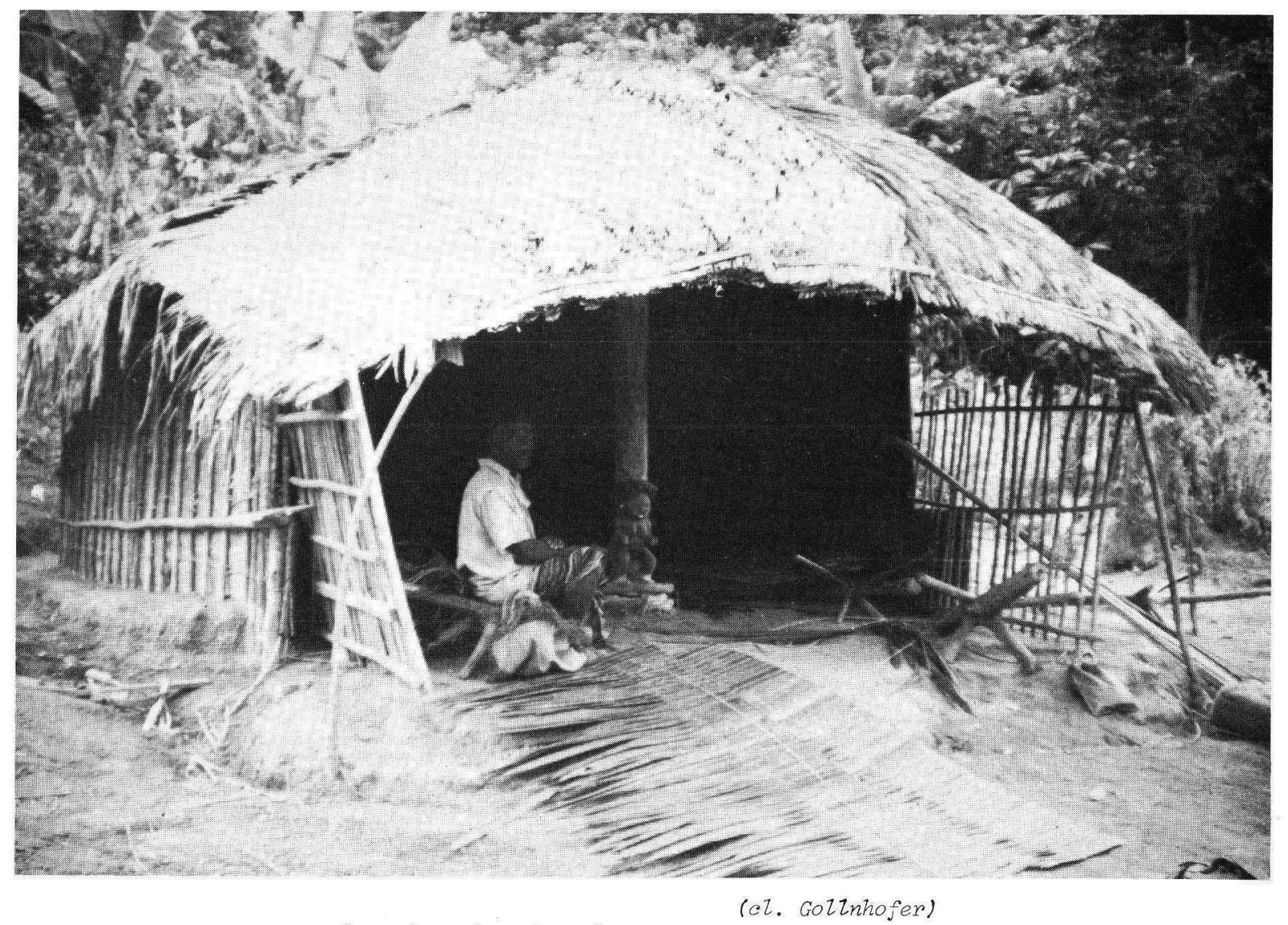

La maison de culte ebanza 
NOTES

(1) Par exemple un fragment du corps de l'éléphant pour acquérir la force de cet animat. ou pour déclencher un éléphantiasis.

(2) Gollnhofer 0 . et Sillans R., Aperçu sur les pratiques sacrificielles chez les Mitsogho, Systèmes de pensée en Afrique Noire, C.N.R.S., Cahier 4, 1972, pp. 167-174.

(3) Les mighangha, qui relèvent de la médication psychique dans un contexte rituel sont complétés par les bogha, qui correspondent à la médication somatique (Gollnhofer 0 , et Sillans R., Aspects du phénomène de concensus dans la psychothérapie ghetsogho, in La notion de personne en Afrique Noire, C.N.R.S., Paris, 1973, pp. 454-563).

(4) II s'agit des plantes suivantes : muemwemo (espèce indéterminée), kuta (Carpolobia alba Don ou Carpolobia Zutea Don (Polygalacées), ghekadi (Elaeis guineensis Jacq. var. nigrescens A. Chev. (Palmacées), mokange (Guibourtia amoldiana (De Wild. et Dur.) J. Léonard (Cesalpiniacées), obaka (Guibourtia tessmannii (Harms) J. Léonard (Cesalpiniacées), et gheanga (Randia acuminata Bth. (Rubiacées).

(5) Champignon à grand chapeau en forme d'entonnoir, dont on utilise le mycelium souterrain dans divers rites.

(6) Corde de liane qui représente le cordon ombilical.

(7) Dans le cadre de cet article, il ne saurait être question de décrire toutes les pratiques rituelles faisant intervenir les crânes d'ancêtres auxquelles ont recours les Mitsogho. Il était fréquent autrefois de calciner les crânes des morts pour se servir de la poudre d'os comme d'une médecine donnant à ses utilisateurs le pouvoir de se rendre invisibles. Les guerriers, avant de partir en expédition, s'enduisaient le front de cette poudre. 